\title{
A novel ABCC2 p.G693R mutation resulting in loss of function of MRP2: a recurrent cause of hyperbilirubinemia in Dubin- Johnson syndrome in China
}

Lina Wu ( $\nabla$ w4932965@163.com )

Beijing Friendship Hospital, Capital Medical University https://orcid.org/0000-0002-3220-6041

\section{Yanmeng Li}

Capital Medical University Affiliated Beijing Friendship Hospital

\section{Yi Song}

Capital Medical University Affiliated Beijing Friendship Hospital

Donghu Zhou

Capital Medical University Affiliated Beijing Friendship Hospital

Siyu Jia

Capital Medical University Affiliated Beijing Friendship Hospital

\section{Anjian Xu}

Capital Medical University Affiliated Beijing Friendship Hospital

Wei Zhang

Capital Medical University Affiliated Beijing Friendship Hospital

\section{Hong You}

Capital Medical University Affiliated Beijing Friendship Hospital

Jidong Jia

Capital Medical University Affiliated Beijing Friendship Hospital

Jian Huang

Capital Medical University Affiliated Beijing Friendship Hospital

\section{Xiaojuan Ou}

Capital Medical University Affiliated Beijing Friendship Hospital

\section{Research}

Keywords: Dubin-Johnson syndrome, adenosine triphosphate-binding cassette subfamily C member 2, multidrug resistanceassociated protein 2, missense mutation, biological function

Posted Date: November 14th, 2019

DOI: https://doi.org/10.21203/rs.2.17212/v1

License: (c) (i) This work is licensed under a Creative Commons Attribution 4.0 International License. Read Full License 


\section{Abstract}

Background Dubin-Johnson syndrome (DJS) is a rare autosomal recessive disorder characterized by predominantly conjugated hyperbilirubinemia that is caused by pathogenic mutations in the adenosine triphosphate-binding cassette subfamily $\mathrm{C}$ member 2 ( ABCC2 ) gene, which encodes multidrug resistance-associated protein 2 (MRP2). However, little is known about the causative mutation of DJS in China. Recently, we have reported a novel ABCC2 p.G693R mutation in two unrelated cases. In the present study, we investigated the pathogenicity of the ABCC2 p.G693R mutation in DJS in China.

Methods Clinical and genetic analysis was conducted for the two patients with the ABCC2 p.G693R mutation. Whole exome sequencing for mutations in other known hyperbilirubinemia-related genes was conducted for the cases with ABCC2 p.G693R. Expression and cellular localization of the mutant MRP2 p.G693R were analyzed by Western blotting and immunofluorescence assay, respectively. Organic anion transport activity was evaluated by the analysis of glutathione-conjugated-monochlorobimane.

Results The two DJS patients with ABCC2 p.G693R mutation, which was conserved among different species, showed typical hyperbilirubinemia phenotype. No pathogenic mutation was identified in the other known hyperbilirubinemia related genes. Functional studies in three cell lines showed that the expression, localization and the organic anion transport activity were significantly compromised by MRP2 p.G693R mutation compared with wild-type MRP2.

Conclusions The ABCC2 p.G693R mutation is associated with loss of function of the MRP2 protein, which may represent one of the major etiological factors of hyperbilirubinemia in DJS in China.

\section{Background}

Dubin-Johnson syndrome (DJS) is an autosomal recessive disorder which was first described in 1954 [1]. As a rare disorder affecting both genders, DJS has been identified in all nationalities and races. Its incidence in Sephardic Jews is approximately 1 in 3,000 [2]. The syndrome is characterized by predominantly conjugated hyperbilirubinemia, which is caused by impairment in the transfer of non-bile acid organic anions from hepatocytes into canaliculi [3].

The adenosine triphosphate-binding cassette subfamily $\mathrm{C}$ member 2 ( $A B C C 2)$ gene, located on chromosome 10q24, encodes the multidrug resistance-associated protein 2 (MRP2). Comprised of 1,545 amino acids, this protein belongs to an integral membrane glycoprotein family [4]. MRP2 traffic from the endoplasmic reticulum to the canalicular membrane of hepatocytes where it functions and then relocalizes back to endosomal vesicles for recycling ${ }^{5}$. This protein is a non-bile acid organic anion transporter and mediates the active transport of conjugate compounds with glutathione or glucuronate from the cytoplasm of hepatocytes into the canaliculi [5].

According to Human Gene Mutation Database (HGMD; www.hgmd.cf.ac.uk), a total of 67 pathogenic genetic mutations in the $A B C C 2$ gene including missense, nonsense, deletions and splice site mutations have been identified in DJS patients. However, no hotspot mutations have been identified in the $A B C C 2$ gene. The majority of the DJS-causing mutations in $A B C C 2$ are related to defects in MRP2 protein synthesis, localization or secretion activities. Some mutations may cause rapid degradation of the mRNA, mislocalization of protein or decreased organic anion transport activity [6]. $A B C C 2$ mutations have been identified in DJS patients worldwide. However, less is known about the causative mutation of DJS in China.

Recently, we identified a novel $A B C C 2$ p.G693R mutation in our previous study [7]. Therefore, in the present study, we investigated the frequency of $A B C C 2$ p.G693R in Chinese DJS patients and examined the pattern and biological consequences of the $A B C C 2$ p.G693R mutation, focusing on their effects on protein maturation, localization and transport activity.

\section{Methods}

\section{Study population}

From the China Registry of Genetic/Metabolic Liver Diseases (ClinicalTrials.gov identifier, NCT03131427), a total of 14 patients suspected with DJS, who had biochemical evidence of fluctuating predominantly conjugated hyperbilirubinemia with or without 
family history, were initially included in the present study between June 2015 and December 2017. Liver biopsy samples were available from 4 of the 14 patients, demonstrating distinctive melanin-like pigment accumulation in hepatocytes and negative immunohistochemical staining for MRP2 in canalicular membrane, which can be confirmed as DJS. Whole blood samples from the 14 patients were collected and stored at $-20^{\circ} \mathrm{C}$ for Sanger sequencing. 7 out of the 14 patients had $A B C C 2$ gene mutations, and 2 of the 7 patients had $A B C C 2$ p.G693R mutation [7].

The study was conducted in accordance with the Declaration of Helsinki. The Ethics Committee of the Beijing Friendship Hospital, Capital Medical University approved the study protocol. All patients provided written informed consent.

\section{Clinical and genetic analysis of the two patients with the $A B C C 2$ p.G693R}

The clinical manifestation of the patients with the $A B C C 2$ p.G693R, including age of onset, duration of jaundice, aggravating or relieving factors was recorded. Past medical history including drug or toxin exposure, alcohol intake, and family history of jaundice or other liver diseases were collected.

Relevant laboratory data of the two patients with the $A B C C 2$ p.G693R were analyzed, including complete blood count, liver function tests, renal function tests and electrolytes, coagulation profile. Abdominal ultrasonography was done to exclude obstruction or dilation of the hepatobiliary tract exist and transient elastography (FibroScan) was conducted to evaluate the liver stiffness.

Conservative analysis was performed by http://genome.ucsc.edu/. Aligned amino acid sequences of human, rhesus, mouse, dog, elephant, chicken, xenopus tropicalis, zebrafish, and lamprey MRP2 with mutation p.G693R loci were analyzed.

\section{Analysis of mutation in other known hyperbilirubinemia genes by whole exome sequencing}

Approximately $1 \mu \mathrm{g}$ of genomic DNA was used to construct a targeted exome library with an insert size of 150-200 bp by an exome capture strategy using a GenCap custom exome enrichment kit (MyGenostics, Beijing, China). Paired-end 100 bp raw reads from each enriched library were generated with an Illumina HiSeq 2000 platform (Illumina, San Diego, USA) according to the manufacturer's protocol. The paired-end reads were aligned against NCBI build 37 of the human genome using Burrows Wheeler Aligner. With the GenomeAnalysis Toolkit (GATK4.1.2.0, https://software.broadinstitute.org/gatk/download/), duplicate reads were marked; local indel realignment was performed, and base quality scores were recalibrated for each sample. The identified potential pathogenic variants were confirmed by Sanger sequencing.

Polyphen-2 (http://genetics.bwh.harvard.edu/pph2),, SIFT (https://sift.bii.a-star.edu.sg/) and MutationTaster (http://www.mutationtaster.org/) were used to predict the biofunctional consequence of the identified variants.

\section{Functional analysis of $A B C C 2$ p.G693R mutant}

Construction of the $A B C C 2$ p.G693R mutant

To create the $A B C C 2$ wild-type plasmid, we amplified $A B C C 2$ from human CDNA and cloned it into Hind III/Not Isites of the pcDNA3.1 vector [8]. The ABCC2 p.G693R construct was generated using the Gene Tailor Site-Directed Mutagenesis System (Invitrogen, Waltham, MA, USA).

Cell culture and transfection 
Human embryonic kidney (HEK) 293A cells and human liver cancer cell lines Huh-7 and HepG2 were obtained from the Cell Resource Center of the Chinese Academy of Medical Science (Beijing, China). The Cell lines were cultured in Dulbecco's modified Eagle's medium supplemented with $10 \%$ fetal bovine serum, 100 units $/ \mathrm{ml}$ penicillin and $100 \mathrm{units} / \mathrm{ml}$ streptomycin. Then the cells were transfected with plasmids expressing ABCC2 wild-type or $A B C C 2$ p.G693R by Lipofectamine 3000 (Invitrogen, Carlsbad, USA) according to the manufacturer's instructions; the culture medium was changed at $6 \mathrm{~h}$ after transfection. Finally, the cells were harvested 24 or $48 \mathrm{~h}$ after transfection.

Measurement of MRP2 expression by Western blotting

The cells were lysed in RIPA buffer with proteinase inhibitor. After centrifugation, the supernatant was used for western blot analysis. Proteins were separated by SDS-PAGE (8\%) and transferred to nitrocellulose membranes; the membranes were incubated with anti-MRP2 monoclonal antibodies M2III-6 (1:200; sc-59608; Santa Cruz Biotechnology, Dallas, TX; a mouse monoclonal antibody raised against a C-terminal region of MRP2 of human origin) or anti-GAPDH antibodies (1:5000; Santa Cruz Biotechnology) overnight at $4 \mathrm{C}$, followed by incubation with horseradish peroxidase (HRP)-conjugated goat anti-mouse antibody (1:5000 dilution; Santa Cruz Biotechnology) for $1 \mathrm{~h}$ at 37C. Immunocomplexes on the membrane were visualized using Immobilon Western Chemiluminescent HRP Substrate (Millipore, Billerica, USA) and Image Lab Software (BIO-RAD, Hercules, USA).

Measurement of subcellular localization by indirect immunofluorescence staining

Immunofluorescence analysis was performed as described previously [9]. The cells were incubated with a primary antibody directed against rabbit anti-MRP2 (ab172630; Abcam) at 4C overnight. After three washes with phosphate-buffered saline for 5 min each, the cells were incubated with anti-rabbit Alex 647-conjugated secondary antibodies (1:200; Invitrogen) for $1 \mathrm{~h}$ at room temperature. Then the cells were visualized and photographed using an FV 300 confocal microscope (Olympus, Tokyo, Japan).

Measurement of organic anion transport activity by export of glutathione conjugated monochlorobimane (GS-MCLB) assay

MCLB is an organic anion transport substrate for MRP2 and has absorption/emission maximal 394/490 nm. MCLB (M1381MP, Thermo, USA) transport study was conducted as described by Terlouw et al [10]. The cells were pre-incubated with $0.2 \mathrm{mmol} / \mathrm{L}$ MCLB in medium for 30 min on ice. The medium was replaced with fresh Hank's medium and incubated at $37^{\circ} \mathrm{C}$. At different time points, medium was collected and the GS-MCLB in the medium was measured by the fluorescence method with a spectrophotometer.

\section{Statistical analysis}

All experiments were carried out at least three times. Statistical analyses were performed using SPSS V12.0 software. Results were expressed as mean \pm standard deviation (SD). Continuous variables were analyzed using the Student's test. A two-sided P value of $<0.05$ was considered statistically significant.

\section{Results}

\section{Clinical and genetic profiles of the DJS patients with $A B C C 2$ p.G693R mutation}

For the two unrelated patients with DJS, patient No. 1 (female, 21 years old) and patient No. 2 (male, 39 years old) harbored the heterozygous allelic variant c. $2190 \mathrm{G}>\mathrm{A}$ in exon 16 of $A B C C 2$, whichresulted in the substitution of arginine for glycine at position 693 (p.G693R) (Fig. 1a). Sequence comparison showed that amino acid 693 of MRP2 was conserved among different species (Fig. 1b). The two patients harbored the variant of p.G808V or p.R529Q in another allele respectively.

The clinical features of these two patients are shown in Table 1. Total bilirubin and direct bilirubin were $94 \mu \mathrm{mol} / \mathrm{L}$ and $54 \mu \mathrm{mol} / \mathrm{L}$ in patient No.1, and $97 \mu \mathrm{mol} / \mathrm{L}$ and $49 \mu \mathrm{mol} / \mathrm{L}$ in patient No.2 respectively. The aminotransferase, alkaline phosphatase, $\gamma^{-}$ glutamyl transpeptidase, total bile acid and albumin in liver function tests were within the normal range. Abdominal 
ultrasonography showed no obstruction or dilation of the hepatobiliary tract in both patients. Liver stiffness by FibroScan was 3.5 Kpa in patient No.1, but not available in patient No. 2.

Results from whole exome sequencing revealed additional p.R110Q (c.329G>A) mutation in the gene 3ß-hydroxysteroid dehydrogenase type 7 (HSD3B7),, which is related with bile acid synthesis type l, in patient No. 2 with ABCC2 p.G693R mutation, with allele frequency of $H S D 3 B 7$ p.R110Q is $5.80 \mathrm{E}-05$ in East Asian and 3.00E-04 in total, respectively.. However, software prediction demonstrated that the missense mutation HSD3B7p.R110Q is benign or tolerable. In patient No. 1 with ABCC2 p.G693R mutation, whole exome sequencing revealed no mutations in the other known hyperbilirubinemia-related genes (Supplementary Table 1).

\section{MRP2 expression was decreased in cell lines stably expressing MRP2 p.G693R}

Western blotting for MRP2 in HEK293, Huh-7 and HepG2 cell lines transfected with vector expressing ABCC2 with the p.G693R mutation demonstrated a decreased expression of theMRP2 p.G693R mutant, probably due to the degradation of the mutant protein, compared with wild-type MRP2 (Fig. 2a).

\section{MRP2 p.G693R mutant was predominantly retained in the cytoplasm rather than the cell surface}

Indirect immunofluorescence staining in HEK293A, Huh-7 and HepG2 cell lines showed that wild-type MRP2 predominantly localized to the cell surface and cytoplasm (Fig. 2b). In contrast, we observed mislocalization of the MRP2 p.G693R mutant, which was predominantly retained in the cytoplasm rather than the cell surface.

\section{MRP2 p.G693R mutant exhibited decreased organic anion transport activity}

Following pre-incubation with MCLB, which was conjugated with glutathione to produce GS-MCLB, the GS-MCLB efflux in HEK293A, Huh-7 and HepG2 cells lines which stably expressed wild-type MRP2 was markedly increased compared with negative control cells $(p<0.05)$ (Fig. 3). However, the efflux of GS-MCLB in cells expressing MRP2 p.G693R was similar to the negative control. These results indicated that the p.G693R mutant exhibited significantly decreased organic anion transport activity compared with the wild-type MRP2.

\section{Discussion}

In the present study, we reported on the clinical manifestations and biological functional consequences of the $A B C C 2$ p.G693R mutation, which was identified with a high frequency of $28.6 \%(2 / 7)$ in a small cohort of Chinese patients with DJS. Functional studies indicated significantly decreased expression, mislocalization, and decreased organic anion transport activity of mutant MRP2 compared with wild-type MRP2, indicating that this variant resulted in loss of function of the MRP2 protein.

DJS is characterized by predominantly conjugated hyperbilirubinemia in liver function tests and black liver in liver biopsies [11]. Urinary coproporphyrin levels, bromsulphalein excretion test and hepatobiliary scintigraphy with iodopanoic acid provide effective methods for identification and diagnosis on DJS $[12,13]$. However, most of the diagnostic tests, including urinary corproporphyrin or bromsulphalein excretion test, are not widely available in clinical practice in China. Diagnose of DJS relies of $A B C C 2$ gene Sanger sequencing or liver histopathology manifestations. Since liver biopsies are invasive, genetic analysis of peripheral blood samples is important. To date, a total of 67 ABCC2 mutations have been reported in DJS according to HGMD. Of the 67 mutations, $62.7 \%(n=42)$ are missense/nonsense mutations, $16.4 \%(n=11)$ comprise small deletions, $7.5 \%(n=5)$ are splice site mutations 
and $4.5 \%(n=3)$ are gross deletions, and the mutations occur throughout the gene. Therefore, identification and functional analysis of causative $A B C C 2$ gene mutations for patients suspected with DJS are essential in diagnosis of DJS in China.

In the present study, whole exome sequencing revealed additional HSD3B7p.R110Q mutation in a case with $A B C C 2$ p.G693R mutation, but no mutations in the other known hyperbilirubinemia-related genes in the other case with $A B C C 2$ p.G693R mutation. Gene HSD3B7, located on chromosome 16p11.2, encodes an enzyme which is a member of the short-chain dehydrogenase/reductase superfamily and involved in the initial stages of the synthesis of bile acids from cholesterol [14]. Pathogenic mutations in $H S D 3 B 7$ are associated with congenital bile acid deficiency type $\mathrm{l}$, which is a life-threatening liver disease and manifests as hyperbilirubinemia and neonatal cholestasis $[15,16]$. However, in silico analysis showed this variant was benign, indicating it might be common variant but not a pathogenic mutation. Thus, the two cases with $A B C C 2$ p.G693R heterozygous mutation showed that the $A B C C 2$ mutation might be the main genetic factor of DJS in China.

In the present study, the p.G693R mutation was observed in 2 out of 7 patients, indicating that the p.G693R mutation might be a potential hotspot mutation in Chinese DJS patients. ABCC2 p.G693R is a missense mutation located in the first ATP-binding domain of MRP2 protein [17]. We demonstrated that while wild-type MRP2 predominantly localized to the cell surface and cytoplasm, the MRP2 p.G693R mutant was predominantly retained in the cytoplasm rather than the cell surface. The mislocalization of the p.G693R mutant may likely be due to deficient maturation and sorting, causing impaired insertion trafficking from the endoplasmic reticulum to the canalicular membrane in hepatocytes, similar to the p.R768W and p.W709R mutants reported in previous studies $[18,19]$.

Furthermore, efflux of GS-MCLB uptake into plasma from cells expressing the p.G693R mutant was markedly reduced compared with cells expressing wild-type MRP2, suggesting that the organic anion transport activity of p.G693R MRP2 on the canalicular membrane was defective [13]. Similarly, Hashimoto et al. reported that p.Q1382R MRP2 impairs substrate-induced ATP hydrolysis, which lead to the defect of organic anion transport activity [6].

We understand that the number of cases analyzed in the current study was limited, and thus more cases with DJS are required to confirm these conclusions. Using the China Registry of Genetic/Metabolic Liver Diseases, we will conduct further genetic and functional studies of DJS to strengthen the relationship between the genotype and phenotype of DJS.

In conclusion, here we show that the $A B C C 2$ p.G693R mutation causes dysfunction of the MRP2 protein, leading to hyperbilirubinemia in DJS, which may represent one of the major etiological factors of hyperbilirubinemia in DJS in China.

\section{Declarations}

\section{Abbreviations}

DJS: Dubin-Johnson syndrome; $A B C C 2$ : Adenosine triphosphate-binding cassette subfamily C member 2; MRP2: Multidrug resistance-associated protein 2; HGMD: Human Gene Mutation Database; HEK: Human embryonic kidney; HRP: Horseradish peroxidase; GS-MCLB: Glutathione conjugated monochlorobimane; SD: Standard deviation; HSD3B7:3ß-hydroxysteroid dehydrogenase type 7

\section{Ethics approval and consent to participate}

\section{Not applicable}

\section{Consent for publication}

\section{Not applicable}




\section{Availability of data and materials}

All the data were collected from the hospital information system and can be available from the corresponding author upon reasonable request.

\section{Competing interests}

The authors declare that they have no competing interests.

\section{Funding}

\section{This work was supported by grants from the Digestive Medical Coordinated Development Center of Beijing Hospitals Authority (No.XXT06).}

\section{Author contributions}

LW collected and analyzed the clinical data, and drafted the article. YL performed the functional analysis of $A B C C 2$ p.G693R mutant. XO, $\mathrm{HJ}$ and $\mathrm{JJ}$ conceived the study and revised the manuscript. YS, DZ, SJ, AX performed the polymerase chain reaction amplification analysis of the $A B C C 2$ gene. WZ and $\mathrm{HY}$ collected the clinical data. All the authors approved the final version of the manuscript.

\section{References}

1.Dubin IN, Johnson FB. Chronic idiopathic jaundice with unidentified pigment in liver cells; a new clinicopathologic entity with a report of 12 cases. Medicine (Baltimore) 1954;33:155-197.

2.Shani M, Seligsohn U, Gilon E, Sheba C, Adam A. Dubin-Johnson syndrome in Israel. I. Clinical, laboratory, and genetic aspects of 101 cases. Q J Med 1970;39:549-567.

3.Togawa T, Mizuochi T, Sugiura T, Kusano H, Tanikawa K, Sasaki T, Ichinose F, et al. Clinical, Pathologic, and Genetic Features of Neonatal Dubin-Johnson Syndrome: A Multicenter Study in Japan. J Pediatr 2018. 10.1016/j.jpeds.2017.12.058.

4.Keppler D. The roles of MRP2, MRP3, OATP1B1, and OATP1B3 in conjugated hyperbilirubinemia. Drug Metab Dispos 2014;42:561-565.

5.Gazzin S, Masutti F, Vitek L, Tiribelli C. The molecular basis of jaundice: An old symptom revisited. Liver Int 2017;37:1094-1102.

6. Hashimoto K, Uchiumi T, Konno T, Ebihara T, Nakamura T, Wada M, Sakisaka S, et al. Trafficking and functional defects by mutations of the ATP-binding domains in MRP2 in patients with Dubin-Johnson syndrome. Hepatology 2002;36:1236-1245.

7.Wu L, Zhang W, Jia S, Zhao X, Zhou D, Xu A, Duan W, et al. Mutation analysis of the ABCC2 gene in Chinese patients with Dubin-Johnson syndrome. Experimental and Therapeutic Medicine 2018; 16:4201-4206.

8.Zhang W, Xu A, Li Y, Zhao S, Zhou D, Wu L, Zhang B, et al. A novel SLC40A1 p.Y333H mutation with gain of function of ferroportin: A recurrent cause of haemochromatosis in China. Liver international 2019;39:1120-1127.

9.Lv T, Zhang W, Xu A, Li Y, Zhou D, Zhang B, Li X, et al. Non- mutations in haemochromatosis in China: combination of heterozygous mutations involving signal peptide variants. Journal of medical genetics 2018;55:650-660. 
10.Terlouw SA, Masereeuw R, van den Broek PH, Notenboom S, Russel FG. Role of multidrug resistance protein 2 (MRP2) in glutathione-bimane efflux from Caco-2 and rat renal proximal tubule cells. British journal of pharmacology 2001;134:931-938.

11.Park SW, Jun CH, Choi SK, Kim HJ, Kim GE. Hepatobiliary and Pancreatic: A black liver of Dubin-Johnson syndrome. J Gastroenterol Hepatol 2018;33:562.

12.Gilibili RR, Chatterjee S, Bagul P, Mosure KW, Murali BV, Mariappan TT, Mandlekar S, et al. Coproporphyrin-I: A Fluorescent, Endogenous Optimal Probe Substrate for ABCC2 (MRP2) Suitable for Vesicle-Based MRP2 Inhibition Assay. Drug Metab Dispos 2017;45:604-611.

13.Erlinger S, Arias IM, Dhumeaux D. Inherited disorders of bilirubin transport and conjugation: new insights into molecular mechanisms and consequences. Gastroenterology 2014;146:1625-1638.

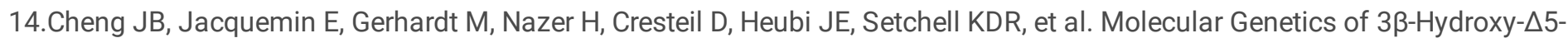
C27-Steroid Oxidoreductase Deficiency in 16 Patients with Loss of Bile Acid Synthesis and Liver Disease. The Journal of Clinical Endocrinology \& Metabolism 2003;88:1833-1841.

15. Huang HY, Zhou H, Wang H, Chen YX, Fang F. Novel Mutations in the 3 $\beta$-hydroxy- $\Delta 5$-C27-steroid Dehydrogenase Gene (HSD3B7) in a Patient with Neonatal Cholestasis. Chinese Medical Journal 2016;129:98-100.

16.Bossi G, Giordano G, Rispoli GA, Maggiore G, Naturale M, Marchetti D, lascone M. Atypical clinical presentation and successful treatment with oral cholic acid of a child with defective bile acid synthesis due to a novel mutation in the gene. Pediatric reports 2017;9:7266.

17.Wen X, Joy MS, Aleksunes LM. In Vitro Transport Activity and Trafficking of MRP2/ABCC2 Polymorphic Variants. Pharm Res 2017;34:1637-1647.

18.Uchiumi T, Tanamachi H, Kuchiwaki K, Kajita M, Matsumoto S, Yagi M, Kanki T, et al. Mutation and functional analysis of ABCC2/multidrug resistance protein 2 in a Japanese patient with Dubin-Johnson syndrome. Hepatol Res 2013;43:569-575.

19.Keppler D. Progress in the Molecular Characterization of Hepatobiliary Transporters. Dig Dis 2017;35:197-202.

\section{Tables}

Table 1 Clinical characteristics and non-synonymous variants identified in DJS patients with $A B C C 2$ p.G693R mutation

\begin{tabular}{|c|c|c|c|c|c|c|c|c|c|c|c|c|c|c|}
\hline $\begin{array}{l}\text { Patient } \\
\text { Number }\end{array}$ & $\begin{array}{c}\text { Age } \\
\text { (year) }\end{array}$ & Sex & Variants & $\begin{array}{c}\text { TB } \\
(\mu \mathrm{mol} / \mathrm{L})\end{array}$ & $\begin{array}{c}\text { DB } \\
(\mu \mathrm{mol} / \mathrm{L})\end{array}$ & $\begin{array}{l}\mathrm{ALT} \\
(\mathrm{U} / \mathrm{L})\end{array}$ & $\begin{array}{l}\text { AST } \\
(\mathrm{U} / \mathrm{L})\end{array}$ & $\begin{array}{l}\text { ALP } \\
(\mathrm{U} / \mathrm{L})\end{array}$ & $\begin{array}{l}\text { GGT } \\
(\mathrm{U} / \mathrm{L})\end{array}$ & $\begin{array}{c}\text { TBA } \\
(\mu \mathrm{mol} / \mathrm{L})\end{array}$ & $\begin{array}{l}\text { ALB } \\
(g / L)\end{array}$ & $\begin{array}{l}\text { PTA } \\
(\%)\end{array}$ & Abdominal ultrasonography & $\begin{array}{c}\text { Liver } \\
\text { stiffness }\end{array}$ \\
\hline
\end{tabular}

\begin{tabular}{|c|c|c|c|c|c|c|c|c|c|c|c|c|c|c|}
\hline 1 & 21 & Female & $\begin{array}{c}\text { p.G693R } \\
\text { (c. } 2190 \mathrm{G}>\mathrm{A}) \text {, } \\
\text { p.R529Q } \\
\text { (c.1586G >A) }\end{array}$ & 94 & 54 & 48 & 28 & 79 & 32 & 2.9 & 45 & 98 & Normal & 3.5 \\
\hline
\end{tabular}

\begin{tabular}{|c|c|c|c|c|c|c|c|c|c|c|c|c|c|c|}
\hline 2 & 39 & Male & $\begin{array}{c}\text { p.G693R } \\
\text { (c. } 2190 \mathrm{G}>\mathrm{A}) \text {, } \\
\text { p.G808V } \\
(\text { c. } 2536 \mathrm{G}>\mathrm{T})\end{array}$ & 97 & 49 & 43 & 17 & 78 & 23 & 2.8 & 46 & 109 & $\begin{array}{c}\text { Hepatic } \\
\text { hemangioma,cholecystolithiasis }\end{array}$ & NA \\
\hline
\end{tabular}

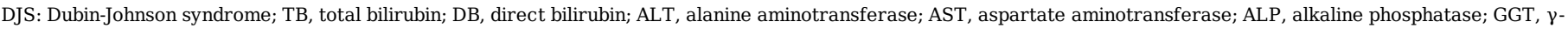
glutamyl transpeptidase; TBA, total bile acid; ALB, albumin; PTA, prothrombin activity; NA, not available. 


\section{Figures}

a

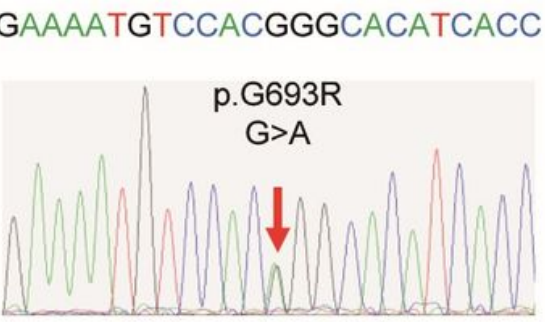

Common mutation
b Human
Rhesus
Mouse
Dog
Elephant
Chicken
X. tropicalis
Zebrafish
Lamprey

\begin{tabular}{|c|c|c|c|c|c|c|c|c|c|c|c|c|}
\hline \multicolumn{13}{|c|}{$A B C C 2 / M R P 2$ protein sequence } \\
\hline L & G & $E$ & $M$ & $E$ & $N$ & $v$ & $\mathrm{H}$ & $G$ & $\mathrm{H}$ & I & $T$ & I \\
\hline L & G & $E$ & $M$ & $E$ & $\mathrm{~N}$ & $V$ & $\mathrm{H}$ & G & H & I & $T$ & I \\
\hline L & G & $E$ & $M$ & $E$ & $\mathrm{~N}$ & V & $\mathrm{H}$ & G & H & I & $T$ & I \\
\hline L & G & $E$ & $M$ & $E$ & D & $V$ & $\mathrm{H}$ & G & $\mathrm{H}$ & I & $T$ & I \\
\hline $\mathrm{L}$ & G & $E$ & $M$ & $E$ & $N$ & V & $\mathrm{H}$ & G & H & I & $T$ & I \\
\hline L & G & $E$ & $M$ & $E$ & $N$ & I & K & G & H & I & $\mathbb{N}$ & I \\
\hline L & G & $E$ & $M$ & D & H & $v$ & $G$ & G & $Y$ & I & $N$ & I \\
\hline L & G & $E$ & L & $\mathrm{H}$ & $\$$ & I & $\mathrm{K}$ & G & $N$ & I & $N$ & I \\
\hline L & G & $\mathrm{E}$ & $M$ & $\mathrm{E}$ & $\mathrm{K}$ & L & $Q$ & G & $T$ & V & ค & $M$ \\
\hline 685 & & & & & 69 & & & 693 & & 695 & & \\
\hline
\end{tabular}

Figure 1

Mutation analysis of the two DJS cases with ABCC2 p.G693R. a Sequencing of the heterozygous missense mutation c.2190G $>A$, p.G693R in exon 16 in both cases. b Aligned amino acid sequences of human, rhesus, mouse, dog, elephant, chicken, xenopus tropicalis, zebrafish, and lamprey MRP2 with mutation p.G693R flanking regions. The position of the p.G693R mutation is indicated by the red box. Alignment was performed by http://genome.ucsc.edu/ 


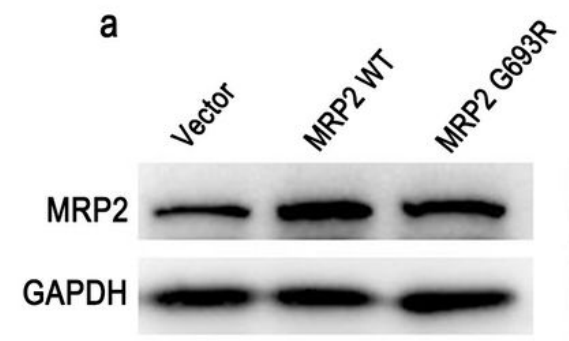

HEK293A

b
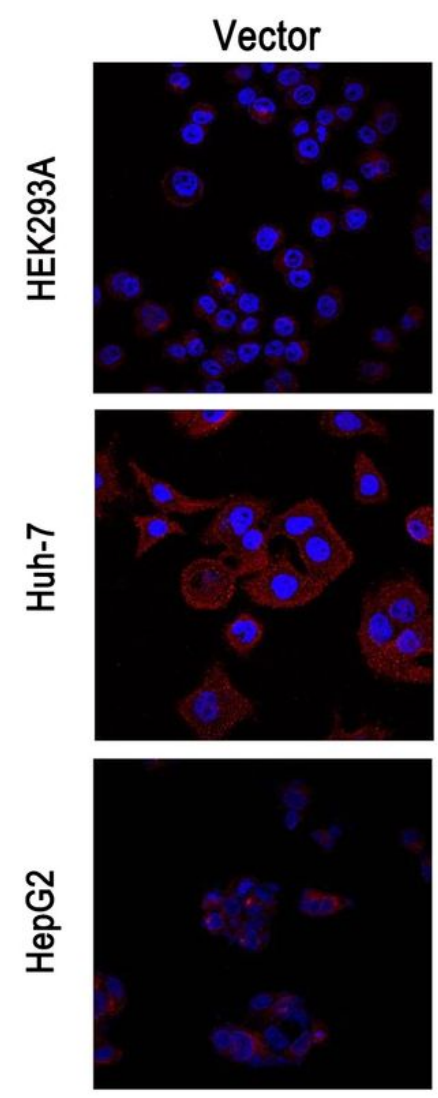
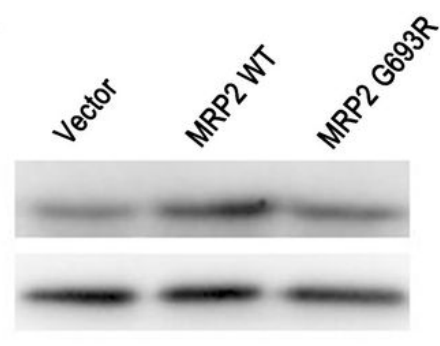

Huh-7

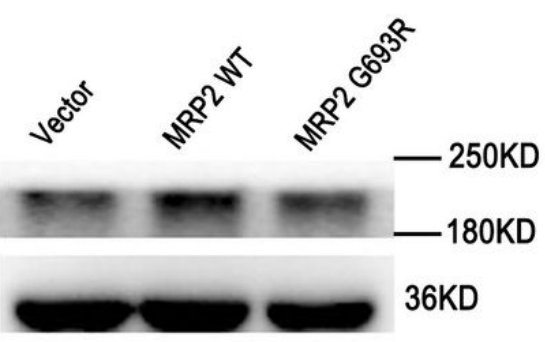

HepG2
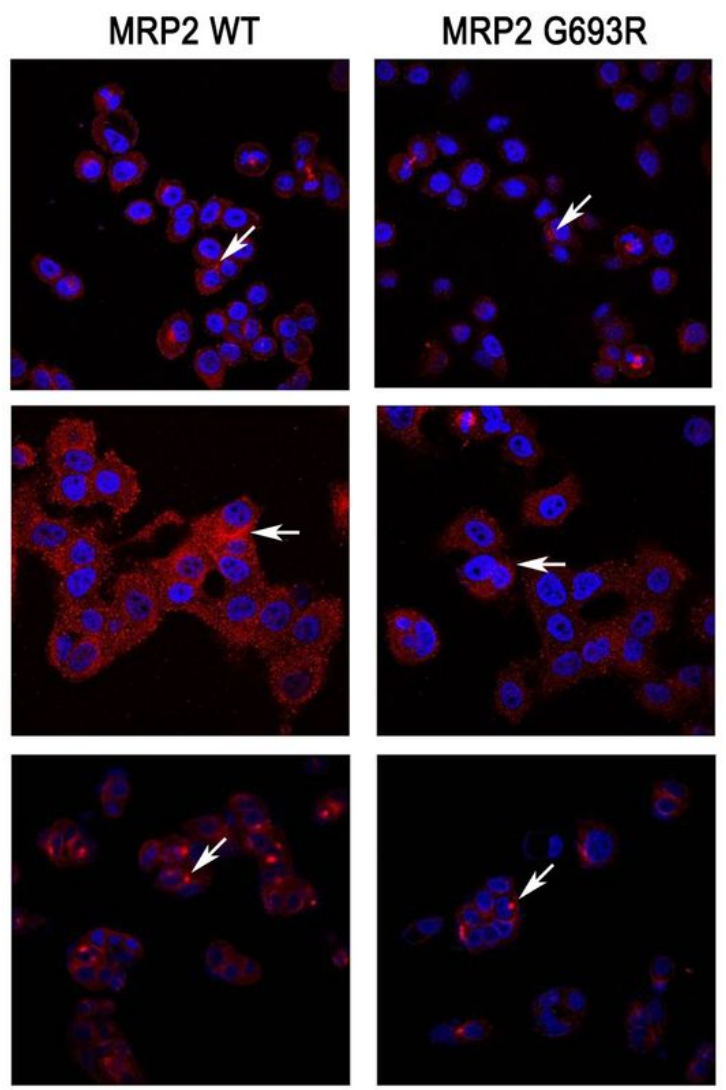

\section{Figure 2}

The MRP2 p.G693R mutant showed decreased expression and mislocalization in three cell lines in vitro. a Western blotting demonstrated significantly decreased expression of MRP2 in cell lines expressing the p.G693R mutant compared with those expressing wild-type MRP2. b Immunofluorescence assay showed the mislocalization of the MRP2 p.G693R mutant, which was predominantly retained in the cytoplasm rather than the cell surface 

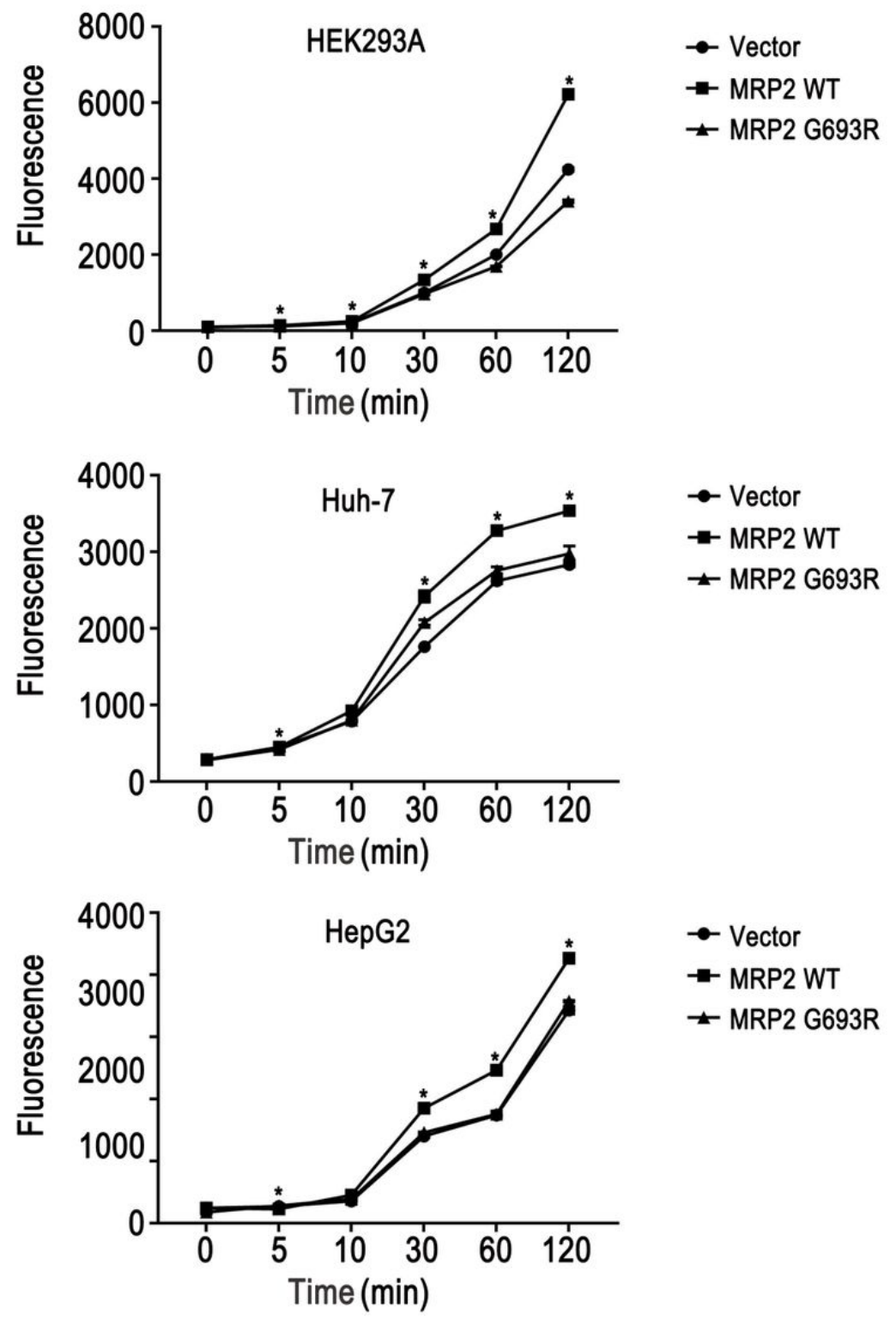

Figure 3

MRP2 p.G693R mutant exhibited decreased organic anion transport activity. Compared with wild-type MRP2, the MRP2 p.G693R mutant exhibited decreased organic anion transport activity in HEK293A, Huh-7 and HepG2 cell lines. *P $<0.05$

\section{Supplementary Files}

This is a list of supplementary files associated with this preprint. Click to download.

- SupplementaryTable1.docx 